\title{
Stability Analysis of Pulse-Width-Modulated Feedback Systems with Time-Varying Delays
}

\author{
Zhong Zhang, Huahui Han, Qiling Zhao, and Lixia Ye \\ College of Mathematics and Statistics, Chongqing University, Chongqing 400044, China \\ Correspondence should be addressed to Zhong Zhang; zhanguicqu@gmail.com \\ Received 3 January 2014; Revised 26 March 2014; Accepted 7 April 2014; Published 27 April 2014 \\ Academic Editor: Chuandong Li
}

Copyright (C) 2014 Zhong Zhang et al. This is an open access article distributed under the Creative Commons Attribution License, which permits unrestricted use, distribution, and reproduction in any medium, provided the original work is properly cited.

\begin{abstract}
The stability problem of pulse-width-modulated feedback systems with time-varying delays and stochastic perturbations is studied. With the help of an improved functional construction method, we establish a new Lyapunov-Krasovskii functional and derive several stability criteria about $p$ th moment exponential stability.
\end{abstract}

\section{Introduction}

PWM has been widely used in many fields, such as attitude control systems, adaptive control systems, signal processing, and modeling of neuron behavior [1-3]. In actual progress, it has always operated in all kinds of disturbances. At the same time, time-varying delays inevitably occur owing to the unavoidable finite switching speed of amplifiers. For some systems, the effect of time-varying delays can be ignored. How to keep the scheduled operation or work of the state running well, especially in engineering applications, is becoming more and more significant.

A growing number of scholars are devoting time to the PWM feedback systems; meanwhile a set of stability results has been established by a variety of methods [4-12]. Also, lots of scholars have researched some systems with delays [7, 13-19]. In [15], the authors investigated robust exponential stability and delayed-state-feedback stabilization of uncertain impulsive stochastic systems with time-varying delays. Sun and Cao [16] gave some definitions on the pth moment exponential stability in mean and established several $p$ th moment globally stability criteria in mean. By using the Lyapunov technique and Razumikhin method, the authors in [17] investigated impulsive effects on stability analysis of high-order BAM neural networks with time delays. And in [18], they established the global exponential stability of the neural networks with its estimated exponential convergence rate. The authors in [19] gave a time-varying delay-dependent criterion for impulsive synchronization to ensure the delayed discrete complex networks switching topology tending to a synchronous state.

To the best of the author's knowledge, there are a few (if any) results for the stability analysis of the critical case of PWM systems with time-varying delays and stochastic perturbations, most of the existing work only considers one condition $[7,8,15]$. In the present paper, we try to make a contribution to this issue. It is noted that the linear plant in this paper has one and only one pole at origin, and the rest of the poles are in the left side of the complex plane, which is more representative and more universal. Based on the references $[7,13]$, the present paper will further study the stability of PWM feedback systems with timevarying delays and establish several new stability criteria. In Section 2, we give some definitions and lemmas. In Section 3, firstly, a criterion on mean square exponential stability of stochastic feedback systems with time-varying delays is given. Secondly, by introducing new variables, we will establish a new Lyapunov-Krasovskii functional with the help of an improved functional construction method. Then, associating with linear matrix inequalities, we will establish criteria for the $p$ th moment exponential stability and the $p$ th moment exponential asymptotic stability. Finally, we demonstrate the applicability of our results by means of an example. 


\section{Notations and Definitions}

A pulse-width modulator is described by

$$
\begin{aligned}
u(t) & =m(e(t)) \\
& = \begin{cases}M \operatorname{sgn}(e(k T)), & t \in\left[k T, k T+T_{k}\right), \\
0, & t \in\left[k T+T_{k}, k T+T\right),\end{cases}
\end{aligned}
$$

with

$$
T_{k}= \begin{cases}\beta|e(k T)|, & |e(k T)| \leq \frac{T}{\beta}, \\ 0, & |e(k T)|>\frac{T}{\beta},\end{cases}
$$

where $e(t)=r(t)-f(t), r(t)$ is the external input and $f(t)$ is the system output and $T_{k}$ is the pulse-width for $k=0,1,2, \ldots$. The sampling period $T$, the amplitude of the pulse $M$, and $\beta$ are all assumed to be constants.

Consider the following stochastic PWM feedback system with time-varying delays:

$$
\begin{aligned}
d x(t)= & A x(t) d t+A_{d} x(t-d) d t+H u(t) d t \\
& +D \vartheta(t, x(t), x(t-d)) d W_{t}, \\
& z(t)=C_{1} x(t)+C_{2} x(t-d),
\end{aligned}
$$

where $x \in R^{n}, u, \vartheta \in R, u$ is the output of the pulsewidth modulator, $\vartheta$ is the nonlinear stochastic perturbations, $A, A_{d}, H, D$ are the matrices of appropriate dimensions, $H$ is the pulse-width control matrix, $0<d \leq T_{k}$, and $W_{t}$ is a scalar wiener process which is defined on the probability space $(\Omega, F, P)$.

Note that $x_{e}=0$ is an equilibrium point of stochastic PWM feedback systems.

Definition 1. Let $(X, d)$ be a metric space, $X \subset R^{n}, A \subset X$, and $T \subset R^{+}$. For any fixed $a \in A$ ( $a$ is called the initial state), $t_{0} \in$ $T$, a stochastic process $\left\{x\left(t, \omega, a, t_{0}\right), t \in T_{a, t_{0}}\right\}$ with domain $X$ is called a stochastic motion if $x\left(t_{0}, \omega, a, t_{0}\right)=a$ for all $\omega \in \Omega$, where $T_{a, t_{0}}=\left[t_{0}, t_{1}\right) \cap T, t_{1}>t_{0}$, and $t_{1}$ is finite or infinite.

Definition 2. Let $S$ be a family of stochastic motions with domain $X$ given by

$$
S \subset\left\{x\left(\cdot, \cdot, a, t_{0}\right): x\left(t_{0}, \omega, a, t_{0}\right)=a, \omega \in \Omega, a \in A, t_{0} \in T\right\} .
$$

We call the four-tuple $\{T, X, A, S\}$ a stochastic dynamical system.

Definition 3 (see $[20,21]$ ). For any fixed functional $V$, the infinitesimal generation operator $\mathscr{L} V(x(t), t)$ is

$$
\begin{aligned}
\mathscr{L} V(x(t), t) \\
\quad=\lim _{\Delta \rightarrow 0^{+}} \frac{1}{\Delta}[E(V(x(t+\Delta), t+\Delta)-V(x(t), t))] .
\end{aligned}
$$

Definition 4 (see $[20,21])$. A stochastic system is $p$ th moment exponential stability. If for any fixed initial condition, there exist $\alpha>0$ and $\beta \geq 1$, then

$$
E\|x(t)\|^{p} \leq \beta \exp (-\alpha t) \sup _{-d \leq \theta \leq 0} E\|\xi(\theta)\|^{p}, \quad \forall t \geq 0 .
$$

Especially, when $p=2$, we called the system mean square exponential stability.

Definition 5 (see $[20,21])$. A stochastic system is pth moment exponential asymptotic stability. If for any fixed initial condition, then

$$
\lim _{t \rightarrow \infty} E\|x(t)\|^{p}=0
$$

Especially, when $p=2$, we called the system mean square exponential asymptotic stability.

Lemma 6 (see $[20,21]$ ). A stochastic system $u(t)=F y(t)$ is mean square stability if there exists a Lyapunov-Krasovskii functional $V(x(t), t)>0$ and its infinitesimal generation operator satisfies $\mathscr{L} V(x(t), t)<0$.

Lemma 7 (see [14]). For any constant matrix $M \in R^{m \times m}, M=$ $M^{T}>0$, scalar $\gamma>0$, and vector function $\omega:[0, \gamma] \rightarrow R^{m}$, the integrations in the following are well defined; then

$$
\gamma \int_{0}^{\gamma} \omega^{T}(\beta) M \omega(\beta) d \beta \geq\left(\int_{0}^{\gamma} \omega(\beta) d \beta\right)^{T} M\left(\int_{0}^{\gamma} \omega(\beta) d \beta\right) .
$$

Lemma 8(a) (see [22] (Schur complement 1)). Given the Hermite matrix $S=\left(\begin{array}{ll}S_{11} & S_{12} \\ S_{12}^{T} & S_{22}\end{array}\right)$, then $S<0$ is equivalent to any one of the following conditions:
(1) $S_{11}<0, S_{22}-S_{12}^{T} S_{11}^{-1} S_{12}<0$,
(2) $S_{22}<0, S_{11}-S_{12} S_{22}^{-1} S_{12}^{T}<0$.

Lemma 8(b) (see $[23,24]$ (Schur complement 2)). Assuming that $A=\left(\begin{array}{ll}A_{11} & A_{12} \\ A_{12}^{T} & A_{22}\end{array}\right)$ is a Hermite matrix, then

(1) $A>0 \Leftrightarrow A_{11}>0, A_{22}-A_{12}^{T} A_{11}^{-1} A_{12}>0$,

(2) If $A_{11}>0$, then $A \geq 0 \Leftrightarrow A_{22}-A_{12}^{T} A_{11}^{-1} A_{12} \geq 0$.

Lemma 9 (see [25] (Gronwall inequality)). If $x(t) \leq h(t)+$ $\int_{t_{0}}^{t} k(s) x(s) d s, t \in\left[t_{0}, T\right)$, where all the functions involved are continuous on $\left[t_{0}, T\right), T \leq+\infty$, and $k(t) \geq 0$, then $x(t)$ satisfies

$$
\begin{array}{r}
x(t) \leq h(t)+\int_{t_{0}}^{t} h(s) k(s) \exp \left[\int_{s}^{t} k(u) d u\right] d s \\
t \in\left[t_{0}, T\right) .
\end{array}
$$

If, in addition, $h(t)$ is nondecreasing, then

$$
x(t) \leq h(t) \exp \left(\int_{t_{0}}^{t} k(s) d s\right), \quad t \in\left[t_{0}, T\right) .
$$




\section{Stability Analysis}

The block diagram of the PWM feedback system (3) is shown in Figure 1.

Now, consider the following stochastic feedback system with time-varying delays:

$$
\begin{aligned}
d x(t)= & A x(t) d t+A_{d} x(t-d) d t+B p(t) d t \\
& +g(t, x(t), x(t-d)) d W_{t},
\end{aligned}
$$

where $x \in R^{n}, p(t)$ is a function of $x(t), x(t-d), g$ is the nonlinear stochastic perturbations, $A, A_{d}, B$ are matrices of appropriate dimensions, $0<d \leq T_{k}$, and $W_{t}$ is a scalar wiener process which is defined on the probability space $(\Omega, F, P)$.

For system (11), we let

$$
g(t) \triangleq g(t, x(t), x(t-d)),
$$

which satisfies that

$$
\operatorname{tr}\left[g^{T}(t) g(t)\right] \leq\left\|G_{1}(x(t))\right\|^{2}+\left\|G_{2}(x(t-d))\right\|^{2},
$$

where $G_{1}, G_{2}$ are constant matrices of appropriate dimensions.

We construct the Lyapunov-Krasovskii functional as follows:

$$
V(x(t), t)=\sum_{i=1}^{5} V_{i}(x(t), t),
$$

with

$$
\begin{aligned}
& V_{1}(x(t), t)=x^{T}(t) P x(t), \\
& V_{2}(x(t), t)=\int_{t-d}^{t} x^{T}(s) R_{1} x(s) d s, \\
& V_{3}(x(t), t)=\int_{t-d}^{t} \int_{s}^{t} x^{T}(\gamma) R_{2} x(\gamma) d \gamma d s, \\
& V_{4}(x(t), t)=2 x^{T}(t) Z \int_{t-d}^{t} x(s) d s, \\
& V_{5}(x(t), t)=\int_{-d}^{0} \int_{-d}^{0} x^{T}(t) Q x(t+\zeta) d \zeta d s .
\end{aligned}
$$

Denoting the parameter set as $\Phi:=\left\{P, R_{1}, R_{2}, Z, Q\right\}$, $P, R_{1}, R_{2}, Z, Q$ are Hermite matrices of appropriate dimensions.

Proposition 10 (see [13]). Lyapunov-Krasovskii functional (14) is positive definite if the parameter set $\Phi$ satisfies

$$
R_{1}>0, \quad R_{2}>0, \quad\left[\begin{array}{cc}
P & Z \\
Z^{T} & d^{-1} R_{1}+Q
\end{array}\right]>0 .
$$

Theorem 11 (see [13]). For a fixed scalar $d>0$, the stochastic system (11) with time-varying delays is mean square exponential stability if there exist scalars $\rho>0, \varepsilon>0$, and

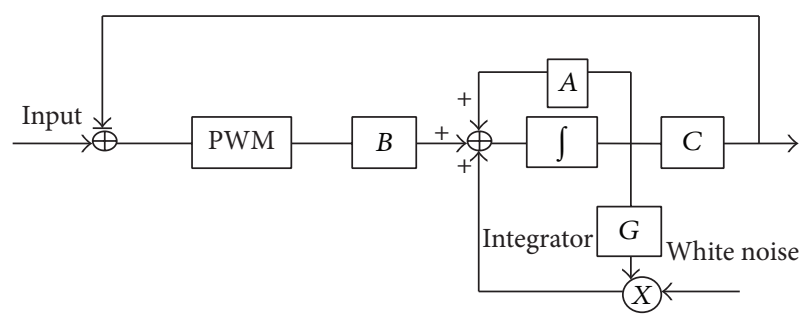

FIGURE 1: Block diagram of PWM feedback systems subjected to multiplicative disturbances.

the parameter set $\Phi$ satisfies Proposition 10, then there exists a linear matrix inequality

$$
\begin{aligned}
\Theta & =\left[\begin{array}{cccc}
\Theta_{11} & P A_{d}-Z+\varepsilon E_{1}^{T} E_{2} & d A^{T} Z+d Q & P B \\
* & -R_{1}+\rho G_{2}^{T} G_{2}+\varepsilon E_{2}^{T} E_{2} & d A_{d}^{T} Z-d Q & 0 \\
* & * & -h^{-1} R_{2} & d Z^{T} B \\
* & * & * & -\varepsilon I
\end{array}\right] \\
& <0
\end{aligned}
$$

where $P \leq \rho I$ :

$$
\begin{aligned}
\Theta_{11}= & P A+A^{T} P+R_{1}+d R_{2}+Z+Z^{T} \\
& +\varepsilon E_{1}^{T} E_{1}+\rho G_{1}^{T} G_{1} .
\end{aligned}
$$

From [13], one obtains that, for $t>0$,

$$
E\|x(t)\|^{2} \leq \frac{\lambda_{\max }(P)+\Delta}{\lambda_{\min }(P)} \sup _{-d \leq \theta \leq 0} E\|\xi(\theta)\|^{2} \exp \left(-\frac{\mu t}{\lambda_{\min }(P)}\right),
$$

where $\Delta=\lambda_{\max }\left(Z^{T} Z\right)+d+\lambda_{\max }\left(R_{1}\right)+d \lambda_{\max }\left(R_{2}\right)+d \lambda_{\max }(Q)$.

Now, let us consider the stochastic PWM feedback system (3) with time-varying delays (Figure 2). After variable substitution, system (3) can be represented as

$$
\begin{aligned}
& d x(t)=A x(t) d t+A_{d} x(t-d) d t+u^{\prime}(t) d t+g^{\prime}(t) d W_{t}, \\
& u^{\prime}(t)=H u(t), \\
& g^{\prime}(t)=G 9(t, x(t), x(t-d)), \\
& z(t)=C_{1} x(t)+C_{2} x(t) .
\end{aligned}
$$

Note that in system (20),

$$
\begin{aligned}
& \left\|u^{\prime}(t)\right\| \leq\left\|M H \sum_{k=1}^{n} \operatorname{sgn}(e(k t))\right\| \leq M\|\lambda H\|, \\
& -n \leq \lambda \leq n,
\end{aligned}
$$

where $n$ is the amount of the PWM period.

By choosing appropriate PWM, we can get $0 \leq \lambda \leq \varepsilon$. Then

$$
\left\|u^{\prime}(t)\right\| \leq \lambda M\|H\|
$$




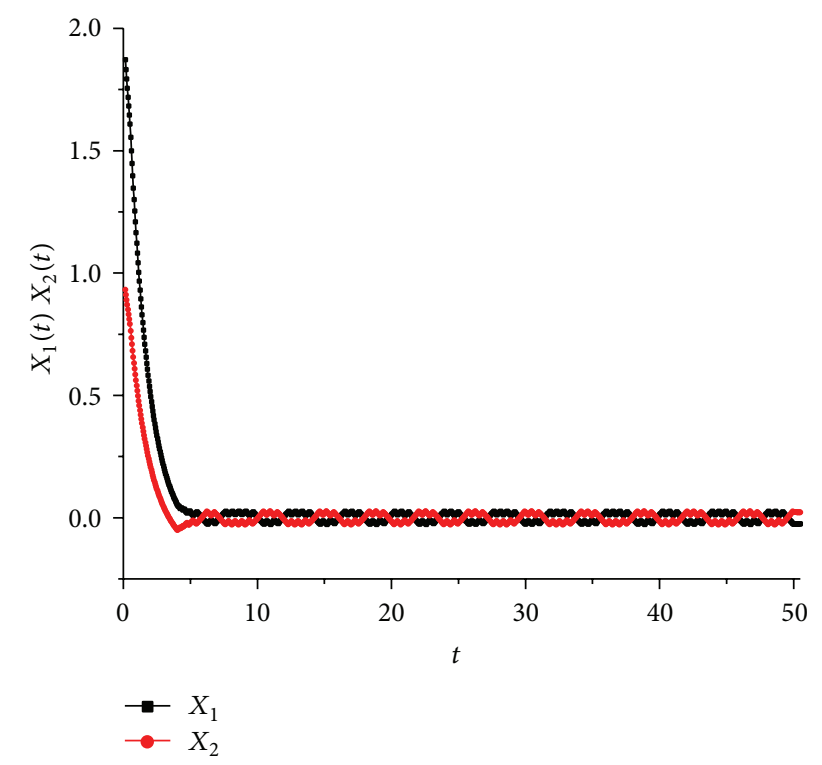

FIGURE 2: Sample response of system (3) with time-varying delays in Example 1 when $M=0.3$.

Associating with (13), we also assume that the stochastic perturbations $g^{\prime}(t)$ satisfy

$$
\operatorname{tr}\left[g^{\prime T}(t) g^{\prime}(t)\right] \leq\left\|D G_{1}(x(t))\right\|^{2}+\left\|D G_{2}(x(t-d))\right\|^{2} .
$$

By simplifying functional (14), we get a new LyapunovKrasovskii functional

$$
V(y(t), t)=\sum_{i=1}^{4} V_{i}(y(t), t),
$$

with

$$
\begin{aligned}
& V_{1}(y(t), t)=y^{T}(t) P y(t), \\
& V_{2}(y(t), t)=\int_{t-d}^{t} y^{T}(s) R y(s) d s, \\
& V_{3}(y(t), t)=2 y^{T}(t) Z \int_{t-d}^{t} y(s) d s, \\
& V_{4}(y(t), t)=\int_{-d}^{0} \int_{-d}^{0} y^{T}(t) Q y(t+\zeta) d \zeta d s .
\end{aligned}
$$

Denoting the parameter set as $\Phi_{1}:=\{P, R, Z, Q\}, R>0$, $y(t)=x^{p / 2}(t), p \in N_{+}, P, R, Z, Q$ are Hermite matrices of appropriate dimensions.

Let functional (24) satisfy Proposition 10, then it is positive definite.

Theorem 12. For time-varying delay $d>0$, the stochastic PWM feedback system (3) with time-varying delays is pth moment exponential stability if there exist scalars $\rho>0, \varepsilon>0$, and the parameter set $\Phi_{1}$ and if the following linear matrix inequality holds:

$$
\Psi=\left[\begin{array}{cccc}
\Psi_{11} & P A_{d}-Z & d A^{T} Z+d Q & P \\
* & -R+\rho D^{T} G_{2}^{T} G_{2} D+\lambda^{2} \varepsilon M^{2} H^{T} H & d A_{d}^{T} Z-d Q & 0 \\
* & * & 0 & d Z^{T} \\
* & * & * & -\varepsilon I
\end{array}\right]<0
$$

where $P \leq \rho I, \Psi_{11}=P A+A^{T} P+R+Z+Z^{T}+\lambda^{2} \varepsilon M^{2} H^{T} H+$ $\rho D^{T} G_{1}^{T} G_{1} D$.

Proof. Using the Itô isometry, we get infinitesimal generation operators of functional (20). Consider

$$
\begin{aligned}
& \mathscr{L} V_{1}(y(t), t) \\
&= \lim _{\Delta \rightarrow 0^{+}} \frac{1}{\Delta}\left[E\left(V_{1}(y(t+\Delta), t+\Delta)-V_{1}(y(t), t)\right)\right] \\
&= 2 y^{T}(t) P\left[A y(t)+A_{d} y(t-d)+u^{\prime}(t)\right] \\
&+\operatorname{Trace}\left[G^{T} W^{T}(t) P W(t) G\right] \\
& \leq 2 y^{T}(t) P\left[A y(t)+A_{d} t(t-d)+u^{\prime}(t)\right] \\
&+\left\|G W_{1}(y(t))\right\|^{2}+\left\|G W_{2}(y(t))\right\|^{2}, \\
& \mathscr{L} V_{2}(y(t), t)=y^{T}(t) R y(t)-y^{T}(t-d) R y(t-d),
\end{aligned}
$$

$$
\begin{aligned}
\mathscr{L} V_{3}(y(t), t)= & 2\left[A y(t)+A_{d} y(t-d)+u^{\prime}(t)\right]^{T} \\
& \times Z \int_{t-h}^{t} y(s) d s \\
& +2 y^{T}(t) Z[y(t)-y(t-d)] \\
\mathscr{L} V_{4}(y(t), t)= & 2 y^{T}(t) Q \int_{t-d}^{t} y(s) d s \\
& +2 y^{T}(t-d) Q \int_{t-d}^{t} y(s) d s .
\end{aligned}
$$

Based on (22), we can get $\lambda^{2} \varepsilon M^{2} H^{T} H-\varepsilon u^{\prime T} u^{\prime} \geq 0$. Then

$$
\begin{aligned}
\mathscr{L} V(y(t), t) & =\sum_{i=1}^{4} \mathscr{L} V_{i}(y(t), t) \\
& \leq \frac{1}{d} \int_{t-d}^{t} \eta^{T}(t, s) \Psi \eta(t, s) d s,
\end{aligned}
$$


where $\eta^{T}(t, s)=\left[\begin{array}{llll}y^{T}(t) & y^{T}(t-d) & y^{T}(s) & u^{\prime T}(t)\end{array}\right]$. Consider

$$
\mathscr{L} V(y(t), t)<0 \quad \text { if } \Psi \text { satisfies } \Psi<0 \text {. }
$$

By Schur complement 1, there exists a scalar $\mu>0$, which makes

$$
\mathscr{L} V(y(t), t) \leq-\mu\|y(t)\|^{2} .
$$

Then, by Dynkin formula, one observes that

$$
\begin{aligned}
& E V(y(t), t)=E V(y(0), 0)+E \int_{0}^{t} \mathscr{L} V(y(s), s) d s, \\
& V_{2}(y(t), t) \leq \lambda_{\max }(R) \int_{-d}^{0}\|y(t+\theta)\|^{2} d \theta, \\
& V_{3}(y(t), t) \leq \lambda_{\max }\left(Z^{T} Z\right)\|y(t)\|^{2}+d \int_{-d}^{0}\|y(t+\theta)\|^{2} d \theta, \\
& V_{4}(y(t), t) \leq d \lambda_{\max }(Q) \int_{-d}^{0}\|y(t+\theta)\|^{2} d \theta .
\end{aligned}
$$

Then

$$
\begin{gathered}
E V(y(0), 0) \leq\left(\lambda_{\max }(P)+\nabla\right) \sup _{-d \leq \theta \leq 0} E\|\xi(\theta)\|^{2}, \\
E V(y(t), t) \geq \lambda_{\min }(P) E\|y(t)\|^{2} \\
\nabla=\lambda_{\max }\left(Z^{T} Z\right)+d+\lambda_{\max }(R)+d \lambda_{\max }(Q) .
\end{gathered}
$$

Thus, by Lemma 9 (Gronwall inequality), we have that

$$
\begin{aligned}
E\|y(t)\|^{2}= & E\left\|x(t)^{p / 2}\right\|^{2} \\
= & E\|x(t)\|^{p} \leq \frac{\lambda_{\max }(P)+\nabla}{\lambda_{\min }(P)} \\
& \times \sup _{-d \leq \theta \leq 0} E\|\xi(\theta)\|^{2} \exp \left(-\frac{\mu t}{\lambda_{\min }(P)}\right), \\
E\|x(t)\|^{p} \leq & \frac{\lambda_{\max }(P)+\nabla}{\lambda_{\min }(P)} \\
& \times \sup _{-d \leq \theta \leq 0} E\|\varsigma(\theta)\|^{p} \exp \left(-\frac{\mu t}{\lambda_{\min }(P)}\right), \\
& \varsigma(\theta)=(\xi(\theta))^{p / 2} .
\end{aligned}
$$

Associating with Definition 4, we know that the system (3) is $p$ th moment exponential stability.

Corollary 13. A stochastic PWM feedback system with timevarying delays which satisfies the conditions of Theorem 12 is pth moment exponential asymptotic stability.

Proof. From the above proof, we obtain

$$
E\|x(t)\|^{p} \leq \frac{\lambda_{\max }(P)+\nabla}{\lambda_{\min }(P)} \sup _{-d \leq \theta \leq 0} E\left\|_{\varsigma}(\theta)\right\|^{p} \exp \left(-\frac{\mu t}{\lambda_{\min }(P)}\right),
$$

where $\lambda_{\max }(p), \lambda_{\min }(p), \nabla, \sup E\|\varsigma(\theta)\|^{p}, \mu$ are all constants. Similarly,

$$
\begin{aligned}
& E\|x(t)\|^{p} \leq K_{1} K_{2} \exp \left(-K_{3} t\right), \\
& \text { where } K_{1}, K_{2}, K_{3} \text { are constants. }
\end{aligned}
$$

Then, we have

$$
\lim _{t \rightarrow \infty} E\|x(t)\|^{p} \leq \lim _{t \rightarrow \infty} K_{1} K_{2} \exp \left(-K_{3} t\right)=0
$$

By Definition 5, the system (3) is pth moment exponential asymptotic stability.

Then, we discuss the functional (24) based on its parameter set.

As the parameter set satisfies $R>0,\left[\begin{array}{cc}P & Z \\ Z^{T} & d^{-1} R+Q\end{array}\right]>0$, by Schur complement 2, we observe that

$$
P>0, \quad d^{-1} R+Q-Z^{T} P^{-1} Z>0 .
$$

Then we can obtain an estimate about matrix $P$, such that

$$
P>0 \cap P<\left(Z^{T}\right)^{-1}\left(d^{-1} R+Q\right) Z^{-1} .
$$

Remark 14 . For system (3), only $H$ can be controlled easily in reality. So we may let $P=H$ and $H$ satisfies formula (38). Then we get another corollary which can be applied in reality.

Corollary 15. For time-varying delay $d>0$, the system (3) is pth moment exponential stability and pth moment exponential asymptotic stability if there exist scalars $\rho>0, \varepsilon>0$, and the parameter set $\Phi_{1}$ such that the following linear matrix inequality holds:

$$
\Psi=\left[\begin{array}{cccc}
\Psi_{11} & H A_{d}-Z & d A^{T} Z+d Q & H \\
* & -R+\rho D^{T} G_{2}^{T} G_{2} D+\lambda^{2} \varepsilon M^{2} H^{T} H & d A_{d}^{T} Z-d Q & 0 \\
* & * & 0 & d Z^{T} \\
* & * & * & -\varepsilon I
\end{array}\right]<0,
$$




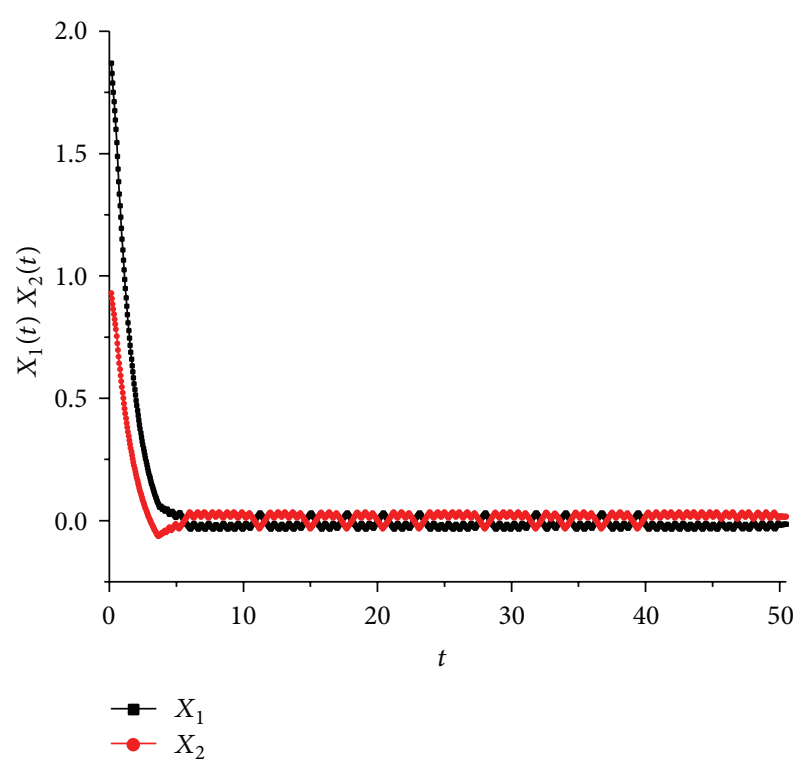

FIGURE 3: Sample response of system (3) with time-varying delays in Example 1 when $M=0.4$.

where $H \leq \rho I, \Psi_{11}=H A+A^{T} H+R+Z+Z^{T}+\lambda^{2} \varepsilon M^{2} H^{T} H+$ $\rho D^{T} G_{1}^{T} G_{1} D$ (Figure 3).

\section{Example}

Example 1. Consider the system (3). Letting $A=\left[\begin{array}{cc}-2 & 0 \\ 0 & -1\end{array}\right]$, $A_{d}=\left[\begin{array}{cc}-1 & 0 \\ 0 & -1\end{array}\right], H=I$, the amplitude of the PWM $M=0.3$, the sampling period $T=1$, and the constant $\beta=1$, the perturbations satisfy $g^{\prime}(t)=\left[\begin{array}{cc}0.02 & 0 \\ 0 & 0.01\end{array}\right]$.

We assume the time accuracy is 0.01 seconds and the system has a tenfold lag; that is, $d=0.1$. And also at the system initial, there is no 0.1 second lag, while it has a 0.1 second lag at system shutdown. The perturbations function on the whole process. For initial-value $x(0)=(2,1)^{T}$, we observe that the system (3) is stable.

By modulating the pulse-width control matrix $H$, we set $M=0.4$. Then, we can get better result of the system (3).

\section{Conclusions}

In this paper, we studied the stochastic PWM feedback systems with time-varying delays and stochastic perturbations. Then, several criteria are established by employing the linear matrix inequality (LMI). Based on the result, an example is presented, which illustrates the effectiveness of the new criteria.

\section{Conflict of Interests}

The authors declare that there is no conflict of interests regarding the publication of this paper.

\section{Acknowledgment}

This paper is supported by the National Natural Science Foundation of China (Grant no. 11202249).

\section{References}

[1] I. Takahashi and H. Mochikawa, "A new control of PWM inverter wave form for minimum loss operation of an induction motor drive," IEEE Transactions on Industry Applications, vol. 21, no. 3, pp. 580-587, 1985.

[2] G. Heinle, "The structure of optimized pulse patterns," in Proceedings of the 5th European Conference on power Electronics and Applications (EPE '93), pp. 378-383, Brighton, UK, 1993.

[3] J. Holtz, "Pulsewidth modulation-a survey," IEEE Transactions on Industrial Electronics, vol. 39, no. 5, pp. 410-420, 1992.

[4] A. Halanaŭ, "Positive definite kernels and the stability of automatic systems," Revue Roumaine de Mathématiques Pures et Appliquées, vol. 9, pp. 751-765, 1964 (Russian).

[5] R. A. Skoog, "on the stability of pulse width modulated feedback systems," IEEE Transactions on Automatic Control, vol. 13, no. 5, pp. 532-538, 1968.

[6] V. M. Kuntesevich and Y. N. Chekhovei, Nonlinear Systems with Pulse Frequency and Pulse Width Modulation, Tekhnika, kiev, Ukranie, 1970.

[7] Z. Zhang and L. Ye, "pth moment exponential stability of stochastic PWM feedback systems with time-varying delays," Abstract and Applied Analysis, vol. 2012, Article ID 796104, 19 pages, 2012.

[8] Z. Zhang and L. Ye, "Moment stability of the critical case of PWM feedback systems with stochastic perturbations," Abstract and Applied Analysis, vol. 2012, Article ID 736408, 19 pages, 2012.

[9] J.-C. Bor and C.-Y. Wu, "Realization of the CMOS pulsewidthmodulation (PWM) neural network with on-chip learning," IEEE Transactions on Circuits and Systems II: Analog and Digital Signal Processing, vol. 45, no. 1, pp. 96-107, 1998.

[10] L. Hou and A. N. Michel, "Stability analysis of pulse-widthmodulated feedback systems," Automatica, vol. 37, no. 9, pp. 1335-1349, 2001.

[11] L. Hou, Qualitative analysis of discontinuous deterministic and stochastic dynamical systems [Ph.D. thesis], University of Notre Dame, 2000.

[12] L. Liu, Y. Shen, and F. Jiang, "The almost sure asymptotic stability and $p$ th moment asymptotic stability of nonlinear stochastic differential systems with polynomial growth," IEEE Transactions on Automatic Control, vol. 56, no. 8, pp. 1985-1990, 2011.

[13] W. Qian, Some issues of systems with time-varying delays [Ph.D. thesis], Zhejiang University, 2009.

[14] K. Gu, "An integral inequality in the stability problem of time-delay systems," in Proceedings of the IEEE Conference on Deeision and Control, pp. 2805-2810, Sydney, Australia, 2000.

[15] P. Cheng, F. Deng, and Y. Peng, "Robust exponential stability and delayed-state-feedback stabilization of uncertain impulsive stochastic systems with time-varying delay," Communications in Nonlinear Science and Numerical Simulation, vol. 17, no. 12, pp. 4740-4752, 2012.

[16] Y. Sun and J. Cao, "pth moment exponential stability of stochastic recurrent neural networks with time-varying delays," Nonlinear Analysis: Real World Applications, vol. 8, no. 4, pp. 1171-1185, 2007. 
[17] C. Li, C. Li, X. Liao, and T. Huang, "Impulsive effects on stability of high-order BAM neural networks with time delays," Neurocomputing, vol. 74, no. 10, pp. 1541-1550, 2011.

[18] C. Li, C. Li, and T. Huang, "Exponential stability of impulsive high-order Hopfield-type neural networks with delays and reaction-diffusion," International Journal of Computer Mathematics, vol. 88, no. 15, pp. 3150-3162, 2011.

[19] C. Li, D. Y. Gao, C. Liu, and C. Guo, "Impulsive control for synchronizing delayed discrete complex networks with switching topology," Neural Computing and Applications, vol. 24, no. 1, pp. 59-68, 2014.

[20] X. Mao, Stochastic Differential Equations and Their Applications, Horwood Publishing, Chichester, UK, 1997.

[21] B. Øksendal, Stochastic Differential Equations: An Introduction with Applications, Springer, New York, NY, USA, 2003.

[22] W. Huang, Schur complement's properties and its application [Ph.D. thesis], Nanjing University of Information Engineering.

[23] S. Wang and Z. Yang, Generalized Inverse Matrix and Its Application, Beijing Industrial University Press, Beijing, China, 1996.

[24] S. Boyd, L. El Ghaoui, E. Feron, and V. Balakrishnan, Linear Matrix Inequalities in System and Control Theory, vol. 15, Society for Industrial and Applied Mathematics, 1994.

[25] H. Ye, J. Gao, and Y. Ding, "A generalized Gronwall inequality and its application to a fractional differential equation," Journal of Mathematical Analysis and Applications, vol. 328, no. 2, pp. 1075-1081, 2007. 


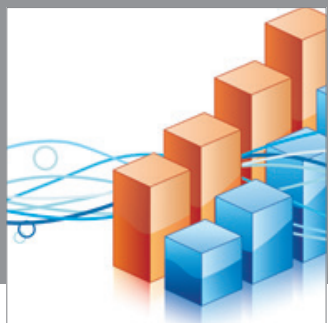

Advances in

Operations Research

mansans

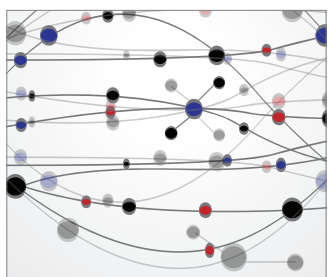

The Scientific World Journal
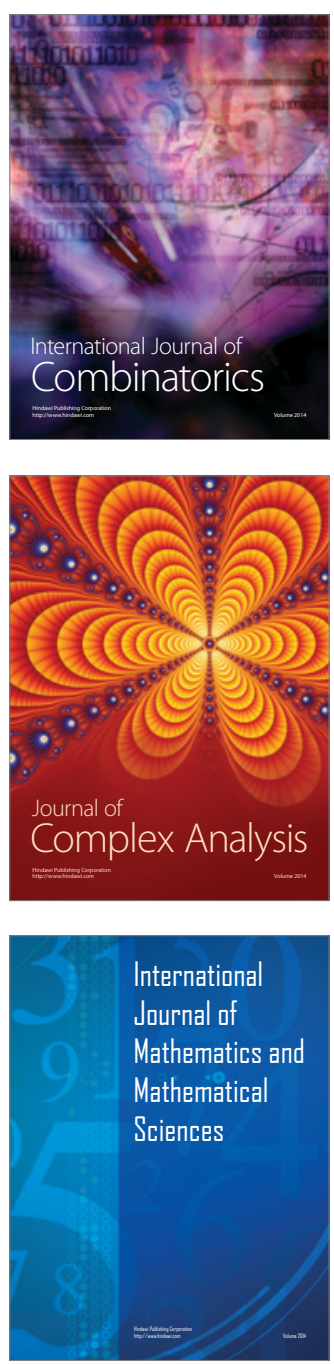
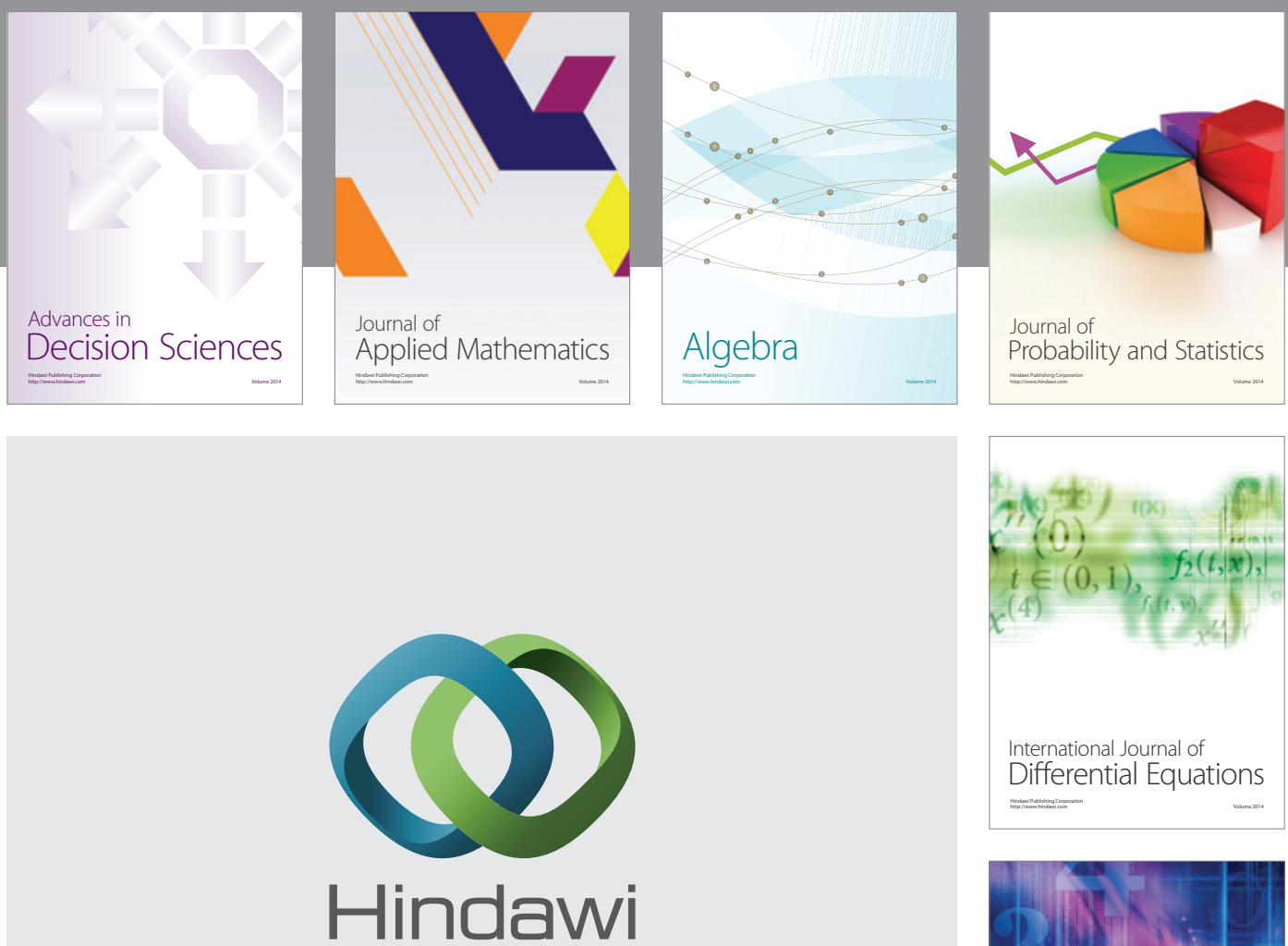

Submit your manuscripts at http://www.hindawi.com
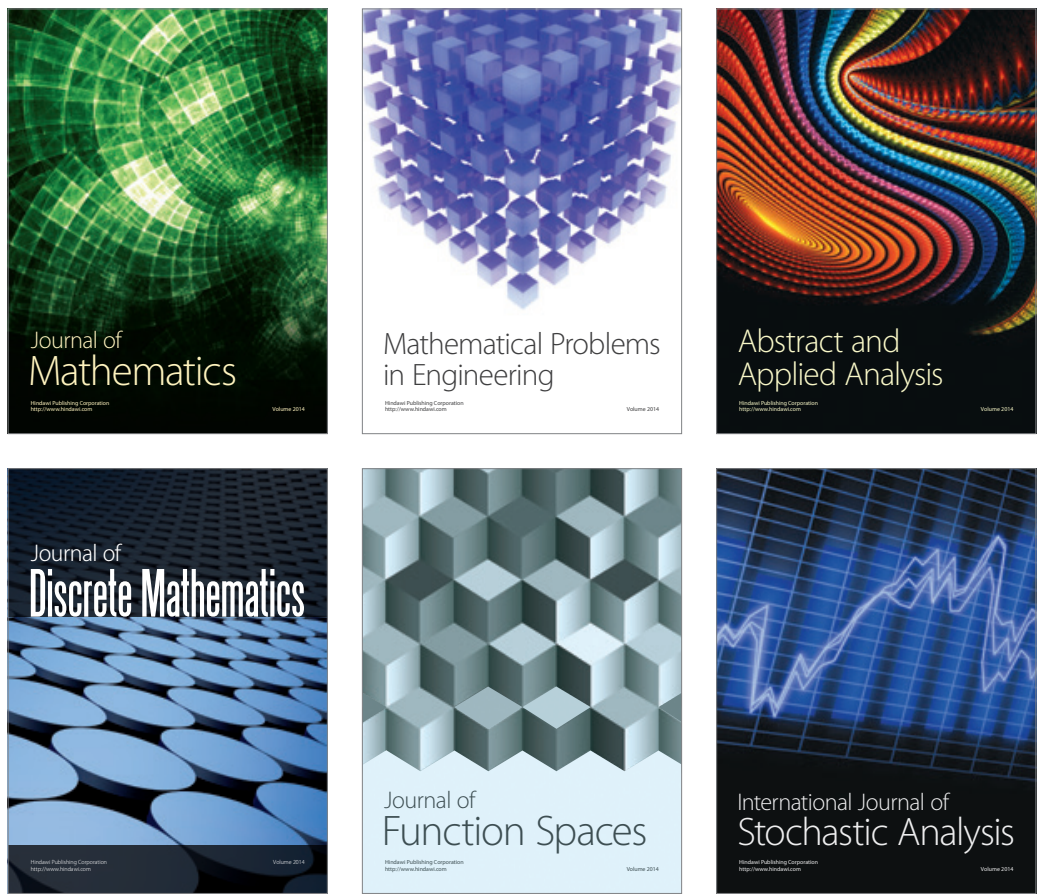

Journal of

Function Spaces

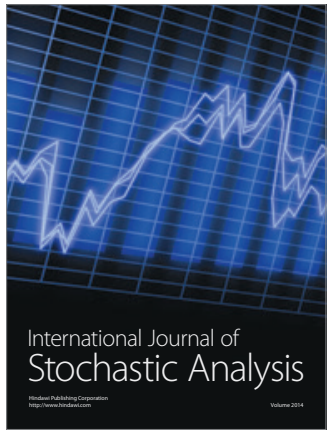

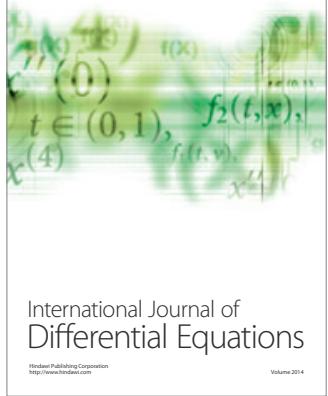
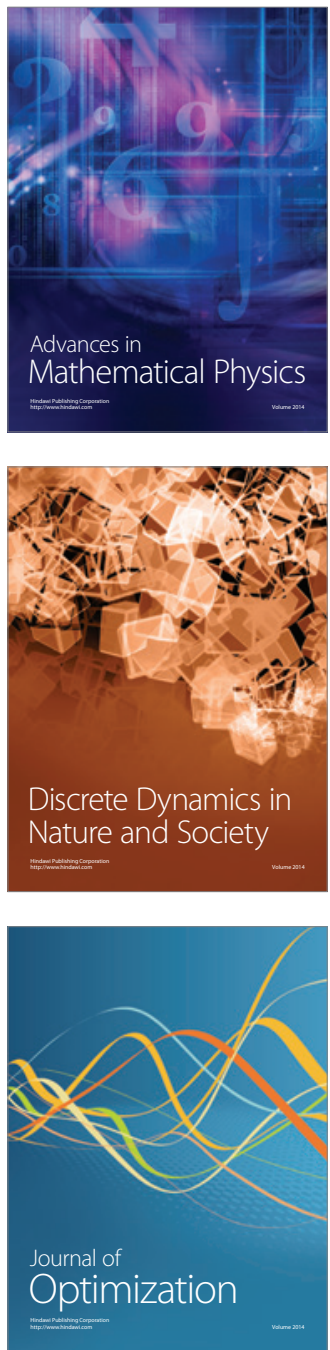